\title{
Anche un uomo può essere entusiasta
}

\author{
Sara Giovine
}

PUBBLICATO: 02 LUGLIO 2019

\section{Quesito:}

Molti lettori ci scrivono per avere delucidazioni sulla corretta declinazione dell'aggettivo entusiasta, oltre che sull'origine e la storia del termine: esiste il maschile singolare entusiasto? Il plurale è entusiaste anche per il maschile o è possibile ricorrere alla forma entusiasti? Quando è riferito a un elemento di genere maschile, l'articolo indeterminativo davanti a entusiasta richiede l'apostrofo? È possibile formare il superlativo assoluto dell'aggettivo?

\section{Anche un uomo può essere entusiasta}

$\mathrm{R}$ ispondiamo subito ai nostri lettori che la forma entusiasto non esiste: al singolare l'aggettivo entusiasta può infatti riferirsi a un sostantivo sia femminile, sia maschile (un giovane entusiasta, una ragazza entusiasta), a differenza del plurale, per cui si distingue invece tra il femminile entusiaste (le bambine entusiaste) e il maschile entusiasti (i bambini entusiasti). Questo perché entusiasta appartiene alla categoria di aggettivi che si declinano solo al plurale (distinguendo tra due forme a seconda del genere), e restano invece invariati al singolare.

Le grammatiche dell'uso (tra cui ricordiamo almeno quella di Serianni ig89 e quella di Renzi-SalviCardinaletti 1995) distinguono infatti gli aggettivi in gruppi (o classi) a seconda della loro modalità di declinazione:

- un primo gruppo comprende gli aggettivi che presentano quattro desinenze, una per ciascun genere e numero, rispettivamente -o/-i per il maschile singolare e plurale e -a/-e per il femminile singolare e plurale (bravo ragazzo/bravi ragazzi, brava ragazza/brave ragazze);

- un secondo gruppo include gli aggettivi variabili solo nel numero, che presentano quindi solo due desinenze, una per il singolare, -e, e una per il plurale, -i, valide per entrambi i generi (storia semplice/storie semplici, quesito semplice/quesiti semplici);

- a un terzo raggruppamento appartengono gli aggettivi invariabili sia nel genere sia nel numero, che presentano una sola desinenza: si tratta in particolare di molti aggettivi di colore, come amaranto, lilla, rosa, viola (giacca/giacche rosa, vestito/vestiti viola); di pari e dei suoi composti (impari, dispari); delle forme composte da un aggettivo di colore e da un aggettivo o un sostantivo che qualificano il colore (come rosso fuoco, verde bottiglia, castano chiaro, ecc.); degli aggettivi di origine straniera non assimilati morfologicamente alla nostra lingua (come beige, chic, snob, standard) e di quelli che si riferiscono a etnie non europee (per esempio bantu, maya, zulu);

- una quarta categoria, che alcune grammatiche ritengono un semplice sottogruppo della prima, è infine formata dagli aggettivi che presentano una sola desinenza per il singolare $(-a)$, comune per i due generi, e due per il plurale, una per il maschile e una per il femminile $(-i /-e)$, come appunto il nostro entusiasta: ne fanno parte gli aggettivi in -ista (gesto/persona egoista, scritti marxisti/forze marxiste), in -cida (spray/polvere insetticida, azioni omicide/pensieri omicidi), in -ita (piatto/bevanda vietnamita, comportamenti ipocriti/affermazioni ipocrite), in -ota (risposta/quesito idiota, abito/gonna keniota) e appunto in -asta (reazione/pubblico entusiasta, autori iconoclasti/manifestazioni iconoclaste), oltre ad altri aggetti in -a, come belga (cittadino/cittadina belga, cittadini 
belgi/cittadine belghe).

La forma entusiasta, che come abbiamo visto può essere al singolare sia maschile sia femminile, quando è impiegata al maschile richiede la variante dell'articolo indeterminativo un, senza apostrofo (per esempio "Giulia lo descrive come un entusiasta"), analogamente a quanto avviene per altre parole di genere maschile inizianti per vocale (un uomo, un animale; sull'uso dell'articolo indeterminativo si veda la scheda a cura di Vera Gheno); la variante apostrofata dell'articolo è invece necessaria quando la forma è usata al femminile (per esempio "la mia amica è un'entusiasta").

Quanto all'origine della parola, la consultazione dei principali dizionari etimologici della lingua italiana ci permette di riconoscere in entusiasta ('che, chi prova o rivela intensa partecipazione,

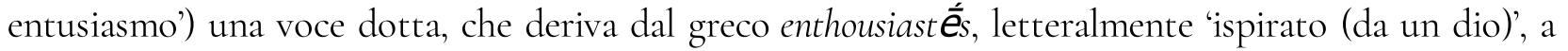
sua volta da enthousiázein 'essere ispirato da un dio' (composto dal prefisso én- 'in, dentro' e da théos 'dio'): nell'antica Grecia la parola enthousiasmós indicava infatti lo stato di esaltazione di chi era invaso e ispirato da una potenza divina, uno stato attribuito in particolare a sacerdoti, indovini e poeti. Secondo la ricostruzione del DELI, con tale significato storico il sostantivo entusiasmo sarebbe stato introdotto in italiano attorno alla metà del XVI secolo, sul modello del francese enthousiasme, e avrebbe in seguito assunto per estensione il significato di intenso sentimento di gioia e partecipazione' con il quale viene oggi comunemente impiegato. Un percorso analogo avrebbe seguito l'aggettivo entusiasta, che, come documenta il DELI, è entrato nell'uso della nostra lingua a partire dal I642 (anch'esso per influsso del corrispettivo francese enthousiaste), ed è stato impiegato prima nel suo originario significato storico di 'chi è invaso dall'entusiasmo, dal furore divino' (che è però oggi ormai desueto) e poi in quello di 'molto soddisfatto, molto lieto'. Inoltre, al plurale la parola (sia come aggettivo, sia come sostantivo) è stata in passato usata per indicare i componenti di gruppi e correnti religiose (quali, per esempio, mormoni, quaccheri e anabattisti) che dichiaravano di essere direttamente ispirati dallo Spirito Santo, ma si tratta di un significato (che rappresenta un'estensione di quello originario) ormai non molto diffuso nell'uso comune.

Infine, per quanto riguarda la possibilità di formare il superlativo assoluto dell'aggettivo entusiasta (in forma sintetica, con l'aggiunta del suffisso -issimo, o in forma analitica, con il ricorso all'avverbio di quantità molto premesso all'aggettivo), le grammatiche precisano come solo gli aggettivi che esprimono una qualità che può essere accresciuta o diminuita possano essere alterati per formare il superlativo (per esempio bello > bellissimo/molto bello, grande > grandissimo/molto grande), mentre non ammettono tale possibilità gli aggettivi che presentano già, nella loro forma base, un significato di tipo elativo, esprimente cioè il grado massimo di intensità di un determinato valore (come immenso, eccellente, straordinario, enorme, ecc.). Anche il significato di entusiasta, pur non potendo essere considerato propriamente elativo, presenta già in sé l'idea dell'intensità e della pienezza (in questo caso di un sentimento di esaltazione e partecipazione): un eventuale ricorso alla forma superlativa risulterebbe di conseguenza ridondante dal punto di vista semantico, e forse anche improprio sul piano grammaticale, sebbene le grammatiche non diano indicazioni esplicite in merito al caso specifico del nostro aggettivo. Unica eccezione la Grammatica di Dardano-Trifone (Dardano-Trifone 1997), che osserva come entusiasta possa essere intensificato con molto, ma non con il suffisso -issimo ("da entusiasta si può avere molto entusiasta, ma non *entusiastissimo") senza però chiarirne le ragioni, limitandosi ad assimilarlo al caso degli aggettivi invariabili, la cui intensificazione può appunto avvenire solo con l'avverbio di quantità ("da blu si può avere molto blu ma non *bluissimo"). Del resto il nostro aggettivo, oltre alla possibilità di essere intensificato con molto, ammette anche il processo inverso, di attenuazione del suo significato, ricorrendo all'avverbio di quantità poco (per esempio "Si è mostrata poco entusiasta all'idea di giocare con me"). In ogni caso, la forma molto entusiasta, seppure discretamente diffusa nell'uso corrente (se ne riscontrano infatti I93.00o occorrenze nelle pagine 
italiane di Google e 136 esempi negli archivi della "Repubblica", uno dei quotidiani più ricettivi nei confronti degli usi linguistici contemporanei), pare propria di un livello di lingua più colloquiale e informale (significativa in tale senso l'assenza di attestazioni nel corpus della Biblioteca Italiana, che raccoglie in rete testi della letteratura italiana dalle origini al 'goo), e sarà quindi preferibile evitarne l'uso almeno nello scritto e in contesti più formali. Grammaticalmente scorretto è invece, come abbiamo detto, il superlativo in -issimo, la cui circolazione risulta di conseguenza decisamente più circoscritta (373 risultati di entusiastissimo e 624 di entusiastissima in rete e, ancora meno quelli relativi al plurale, rispettivamente II 4 di entusiastissimi e appena 25 di entusiastissime; nessuna occorrenza negli archivi dei principali quotidiani nazionali), e limitata a contesti caratterizzati da un uso meno sorvegliato della lingua (come blog, forum e social network), o in cui il ricorso alla forma superlativa risulta motivato da intenti ironico-espressivi, di sottolineatura enfatica del proprio sentimento di gioia e partecipazione. Il suggerimento che forse possiamo dare è quindi di limitarsi all'uso di entusiasta nella sua forma base, di per sé già sufficiente a esprimere l'intensità del sentimento, per evitare errori e non rischiare di tradire un eccesso di... entusiasmo.

\section{Cita come:}

Sara Giovine, Anche un uomo può essere entusiasta , "Italiano digitale", 2019, X, 2019/3 (lugliosettembre)

DOI: $10.35948 / 2532-9006 / 2020.3191$

\section{Copyright 2019 Accademia della Crusca}

Pubblicato con licenza creative commons CC BY-NC-ND 